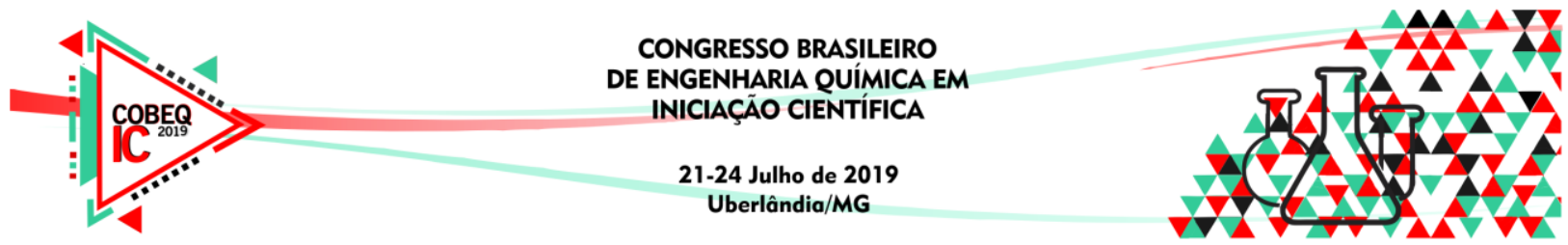

\title{
EFEITO DE LIBERAÇÃO GRADUAL DE NUTRIENTES EM FERTILIZANTE ORGANOMINERAL
}

\author{
B. C. M. HENRIQUE ${ }^{1}$, L. C. M. HENRIQUE ${ }^{2}$ e H. M. HENRIQUE ${ }^{3}$ \\ ${ }^{1}$ Universidade Federal de Uberlândia, Faculdade de Engenharia Elétrica \\ ${ }^{2}$ Universidade Federal de Uberlândia, Faculdade de Engenharia Mecânica \\ ${ }^{3}$ Universidade Federal de Uberlândia, Faculdade de Engenharia Química \\ E-mail para contato: humberto@ufu.br
}

\begin{abstract}
RESUMO - O objetivo deste trabalho foi comprovar cientificamente o efeito de liberação gradual de nutrientes de um fertilizante organomineral em relação ao fertilizante mineral convencional. A metodologia se baseou na medição da solubilização de fósforo e potássio em solução aquosa em função do tempo. O fertilizante organomineral utilizado foi preparado por oclusão com processo de peletização com aditivos. combinado efeito de temperatura, pressão e umidade. Os resultados mostram um efetivo efeito retardador do fertilizante organomineral em relação ao fertilizante mineral.
\end{abstract}

\section{INTRODUÇÃO}

Fertilizante de liberação lenta ou controlada é um fertilizante contendo pelo menos um nutriente que tem sua disponibilidade retardada para uma melhor absorção pelas ou que estende a sua disponibilidade para a planta de maneira significativamente mais duradoura do que um fertilizante de referência de disponibilidade rápida (Trenkel, 2010). Em tais fertilizantes o retardamento da disponibilidade inicial ou período de tempo de disponibilidade prolongada pode ocorrer por uma variedade de mecanismos. Estes incluem a solubilidade controlada em água do material usado nos revestimentos (coating), oclusão, materiais proteicos ou outras formas químicas que sofrem hidrólise lenta produzindo compostos solúveis em água. No caso do fertilizante organomineral utilizado neste trabalho o mecanismo usado foi o de "soldar" uma fração orgânica insolúvel na fração mineral solúvel. O papel de "cola" é desempenhado por uma solução polimérica biodegradável que é adicionada à mistura antes do processo de peletização. As condições operacionais de temperatura, pressão, umidade e granulometria usadas na matriz de peletização permitem que tal solução polimérica "solde" as frações orgânica e mineral. No solo, a fase mineral encontra-se protegida do excesso das águas da chuva uma vez que a matéria orgânica é insolúvel em água e está encapsulando a fração mineral no interior do pellet. Após a solubilização dos minerais presentes na superfície do pellet a "cola" começa a se desfazer por ação da umidade do solo e dos micro-organismos presentes no mesmo e vai liberando gradualmente os minerais presentes no interior do pellet. Com isso, produz-se o tão desejado efeito de liberação gradual de nutrientes. O objetivo deste trabalho foi comprovar cientificamente o efeito de liberação gradual deste fertilizante organomineral. 


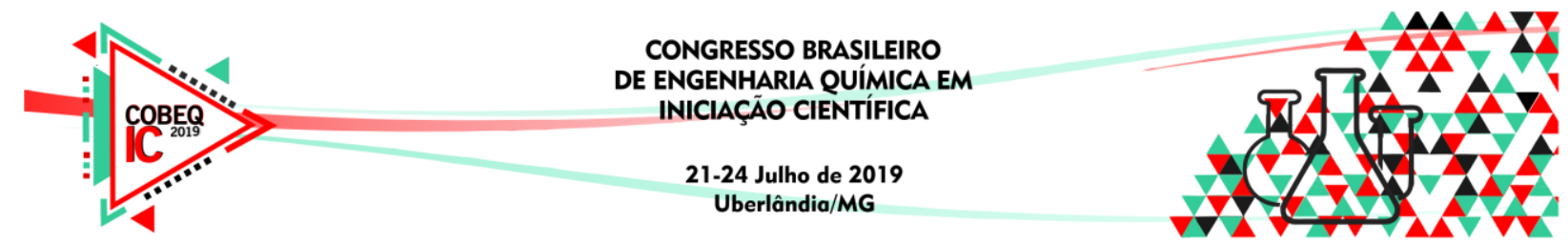

\section{MATERIAIS E MÉTODOS}

A ideia básica por trás deste estudo é medir a quantidade de nutriente solubilizado em uma solução aquosa como uma função do tempo de contato entre o fertilizante e a solução (Henrique, 2010). Os resultados são quantificados em miligramas de nutrientes solubilizado em função do tempo e comparado com uma referência, que no presente caso, são os fertilizantes minerais convencionais. A metodologia é de simples execução e se baseia em tomar uma amostra do fertilizante em estudo de massa e concentração conhecidas. Esta massa é colocada em uma malha de nylon permeável à água, mas impermeável ao fertilizante sólido. Esta massa de fertilizante envolto na malha de nylon é colocada dentro de um frasco de 1.000 $\mathrm{mL}$ de vidro ou de plástico contendo um volume fixo e conhecido de água ( $800 \mathrm{~mL}$ de água). Em seguida, o frasco é colocado em uma incubadora à temperatura constante de $25^{\circ} \mathrm{C} \mathrm{a} 40^{\circ} \mathrm{C}$. No presente caso usou-se a temperatura ambiente de $25^{\circ} \mathrm{C}$. A cada intervalo de amostragem o frasco foi trocado por um novo com igual volume de água. Do frasco usado toma-se uma alíquota de volume conhecido e dosa-se por meios tradicionais o nutriente que foi solubilizado (N, P ou K) e, portanto, passou da fase sólida para a fase líquida. O procedimento é repetido em intervalos de tempo pré-estabelecidos até a solubilização de todo nutriente inicialmente presente na amostra. Os resultados são expressos em porcentagem (\%) da quantidade inicialmente presente na amostra sólida.

\section{RESULTADOS E DISCUSSÃO}

A primeira parte do trabalho foi realizado para estudo da velocidade de liberação de potássio. Foi usado cloreto de potássio (00-00-60) como referência por se tratar da fonte mais usual de $\mathrm{K}$ nos fertilizantes minerais convencionais. Utilizou-se para estudo um fertilizante organomineral na formulação (01-01-20). Utilizou-se massas das amostras dos fertilizantes que continham a mesma quantidade de $\mathrm{mg}$ de $\mathrm{K}_{2} \mathrm{O}$ para que não houvesse interferência no experimento de um menor ou maior gradiente de concentração de $\mathrm{K}_{2} \mathrm{O}$ entre amostra sólida e a solução líquida. Este detalhe é muito importante para efeito de comparação das velocidades de dissolução de amostras de diferentes fertilizantes. No presente caso, ambas as amostras de fertilizante mineral e organomineral continham $11.000 \mathrm{mg}$ de $\mathrm{K}_{2} \mathrm{O} /$ amostra. $\mathrm{O}$ experimento foi realizado com três repetições.

\begin{tabular}{ccc} 
Tabela & \multicolumn{3}{c}{ - Solubilização de $\mathrm{K}_{2} \mathrm{O}$ dos ferti } \\
\cline { 2 - 4 } tempo (h) & Mineral & $\mathrm{OM}$ \\
\hline 0,00 & $0,00 \%$ & $0,00 \%$ \\
0,50 & $46,92 \%$ & $28,71 \%$ \\
1,00 & $69,18 \%$ & $37,82 \%$ \\
1,50 & $92,01 \%$ & $42,36 \%$ \\
3,00 & $98,02 \%$ & $46,15 \%$ \\
9,00 & $98,12 \%$ & $47,31 \%$ \\
24,00 & $98,12 \%$ & $48,57 \%$ \\
\hline
\end{tabular}

Durante as três primeiras horas do experimento o recipiente com água foi trocado a cada 30 minutos. A próxima troca foi realizada depois nove horas do início do experimento e a 


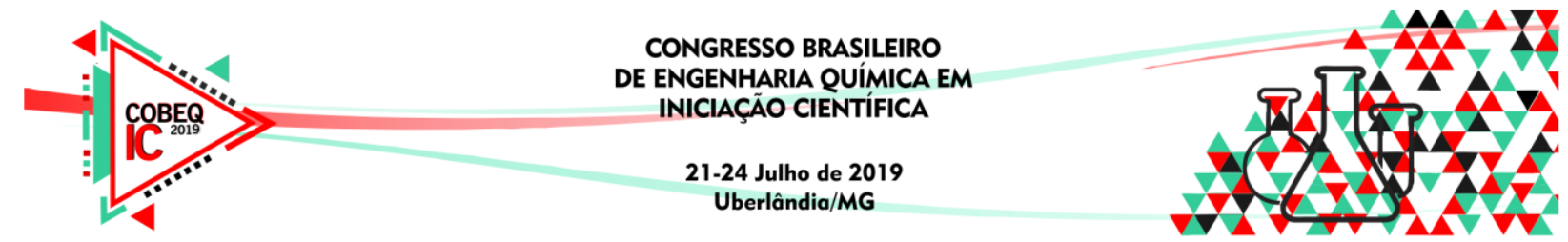

última troca ocorreu há 24 horas após o início do experimento. A Tabela 1 apresenta os resultados da solubilização das amostras de fertilizante mineral e organomineral em 24 horas de experimento. A Figura 1 mostra os mesmos resultados na forma gráfica.

Figura 1 - Curva de liberação de $\mathrm{K}_{2} \mathrm{O}$ dos fertilizantes.

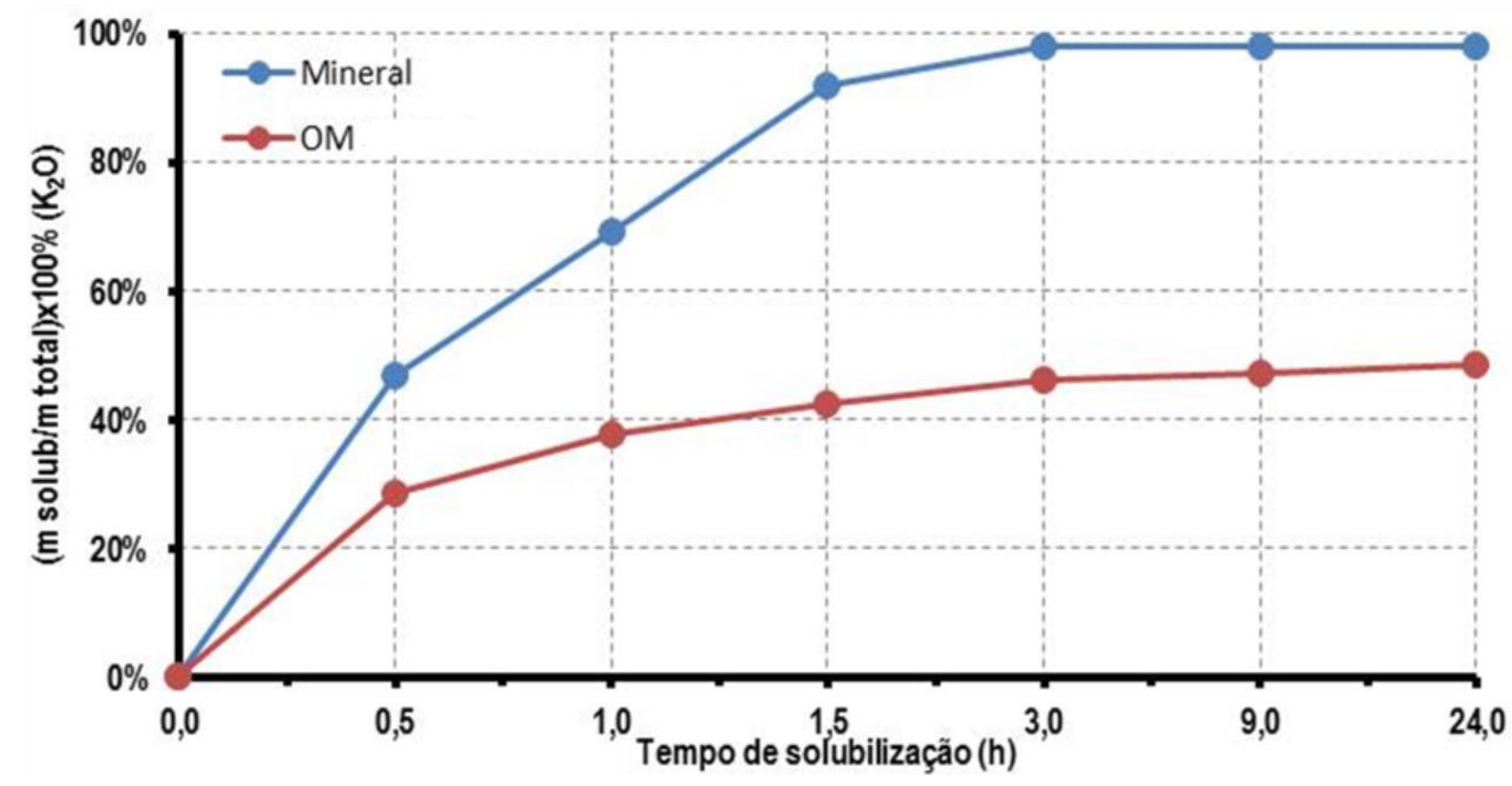

Experimento análogo foi também realizado, mas desta vez com fertilizantes fosfatados. $\mathrm{O}$ objetivo foi determinar a velocidade de dissolução de $\mathrm{P}_{2} \mathrm{O}_{5}$ em ambos os tipos de fertilizantes. $O$ fertilizante mineral utilizado foi fosfato monoamônio (10-52-00) conhecido como MAP e o fertilizante organomineral foi um 05-24-01. As amostras de fertilizante continham $9.500 \mathrm{mg}$ de $\mathrm{P}_{2} \mathrm{O}_{5}$. A Tabela 2 e a Figura 2 mostram os resultados para o fósforo.

Tabela 2 - Solubilização de $\mathrm{P}_{2} \mathrm{O}_{5}$ dos fertilizantes.

\begin{tabular}{ccc}
\hline tempo $(\mathrm{h})$ & Mineral & OM \\
\hline 0,00 & $0,00 \%$ & $0,00 \%$ \\
0,50 & $68,46 \%$ & $35,09 \%$ \\
1,00 & $85,39 \%$ & $48,09 \%$ \\
1,50 & $91,63 \%$ & $56,38 \%$ \\
3,00 & $96,30 \%$ & $66,66 \%$ \\
9,00 & $99,57 \%$ & $74,83 \%$ \\
24,00 & $100,00 \%$ & $77,24 \%$ \\
\hline
\end{tabular}

Os resultados das Tabelas 1 e 2 e das Figuras 1 e 2 revelam claramente que a velocidade de liberação de $\mathrm{K}_{2} \mathrm{O}$ e de $\mathrm{P}_{2} \mathrm{O}_{5}$ foram realizadas de forma bem mais gradual no caso do fertilizante organomineral do que no fertilizante mineral. 


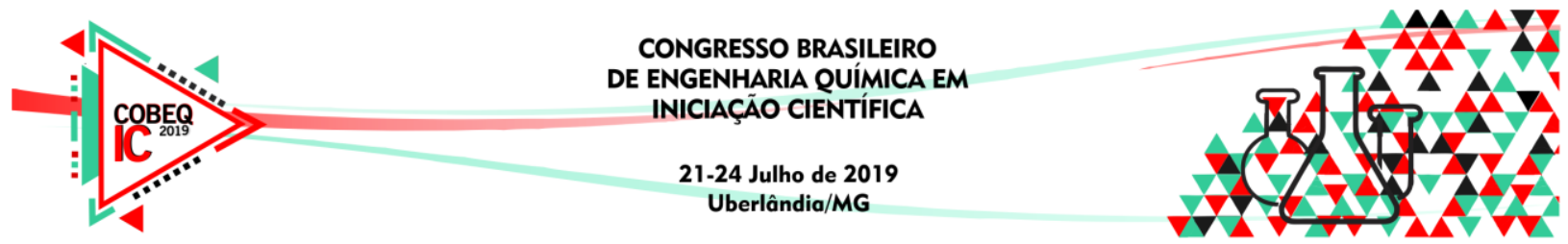

Figura 2 - Curva de liberação de $\mathrm{P}_{2} \mathrm{O}_{5}$ dos fertilizantes.

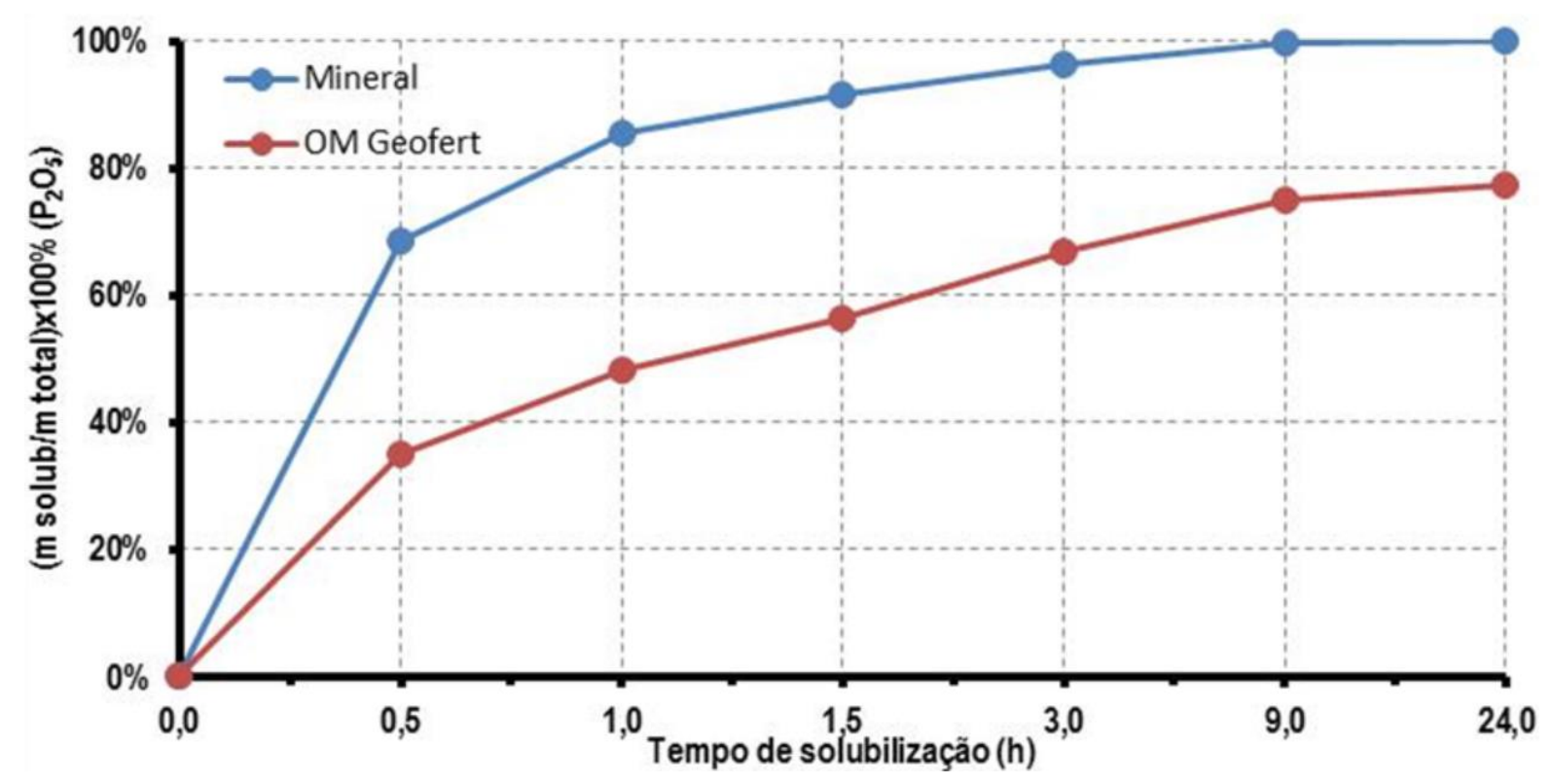

\section{CONCLUSÃO}

Os resultados da Figura 1 e Tabela 1 mostraram claramente que o fertilizante mineral liberou todo seu conteúdo de $\mathrm{K}_{2} \mathrm{O}$ em apenas 3 horas, ao passo que o fertilizante organomineral liberou pouco mais de $46 \%$ de seu conteúdo neste período. Em 24 horas o fertilizante organomineral liberou aproximadamente $49 \%$ de seu conteúdo, mostrando claramente o quão mais gradual é a liberação de $\mathrm{K}_{2} \mathrm{O}$ do fertilizante organomineral em relação ao mineral. Com relação à liberação de $\mathrm{P}_{2} \mathrm{O}_{5}$ chegou-se a resultados semelhantes. Houve liberação de praticamente $100 \%$ do $\mathrm{P}_{2} \mathrm{O}_{5}$ das amostras de fertilizante mineral em apenas 9 horas, enquanto no organomineral houve uma liberação de aproximadamente $75 \%$. Em 24 horas o fertilizante organomineral liberou aproximadamente $77 \%$ de seu conteúdo.

Os resultados apresentados atestam claramente o efeito de liberação gradual de nutrientes do fertilizante organomineral utilizado em relação ao fertilizante mineral convencional. O processo de oclusão da fase mineral do fertilizante com a matéria orgânica em pressão elevada foi capaz de imprimir ao fertilizante este efeito de liberação gradual. A metodologia de teste para a medição da solubilização de fósforo e potássio em solução aquosa em função do tempo se mostrou adequada e fácil implementação.

\section{AGRADECIMENTOS}

À Finep pelos recursos concedidos, à Geociclo Biotecnologia S/A por fornecer os fertilizantes para a realização dos experimentos. 


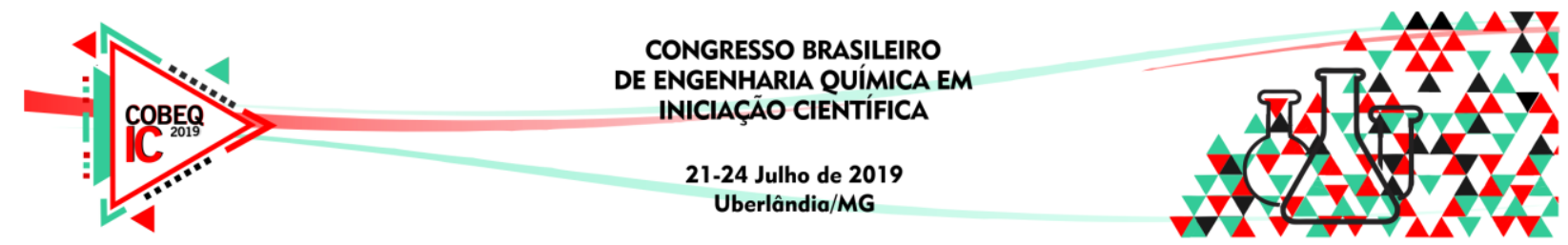

\section{REFERÊNCIAS}

TRENKEL M.E., Slow- and Controlled-Release and Stabilized Fertilizers: An Option for Enhancing Nutrient Efficiency in Agriculture. Second edition, IFA, Paris, France, October 2010.

HENRIQUE, H.M., Geofert: A gênese do fertilizante e seus resultados agronômicos. Relatório Interno. Uberlândia/MG, 2013, 158p. 